\title{
POLIQUETOS BENTÓNICOS INTERMAREALES Y SUBLITORALES DE LA REGIÓN DE AISÉN, CHILE.
}

\author{
INTERTIDAL AND SUBTIDAL BENTHIC POLYCHAETES \\ FROM AISÉN REGION, CHILE.
}

\begin{abstract}
Composition, abundance and richness of the intertidal and shallow subtidal ( $<30$ metres) benthic polychaetes are presented. Several coastal fieldworks in fjords, channels and islands were undertaken between 2001 and 2003 in relationship with Biodiversity Aysen Project. 1.364 individuals and a total number of 76 taxa belonging to 30 families were recorded. The higher species richness was observed by Spionidae and Nereididae with nine and eight species respectively, with Platynereis australis (Schmarda, 1861) showing the highest dominance across overall sampling fieldworks. Specimens of Chrysopetalidae, Saccocirridae and Arenicolidae families are recorded for first time in the region, while another species increase their distribution range. The high species richness of some places (e.g. Kent Island) and some special oceanographic, environmental and geographic conditions suppose that diversity 'hotspots' have place at the Aisen region. A hypothesis is presented in terms of, some polychaetes species with high abundance and frequency in comparison to other invertebrates and specific environmental conditions where they live would allow developing a 'subiotope' in the studied coastal environmental. The 'subiotope' would be mainly defined by dominance of these polychaete species.
\end{abstract}

Key words: Polychaeta, intertidal, shallow waters, biodiversity.

1 Facultad de Ciencias del Mar y de Recursos Naturales, Universidad de Valparaíso. Casilla 5080 Reñaca, Viña del Mar, Chile. eulogio.soto@uv.cl.

2 The Natural History Museum, Zoology Department. Cromwell Road London SW7 5BD United Kingdom. g.paterson@nhm. ac.uk 


\section{RESUMEN}

Se presentan los resultados de composición, abundancia y riqueza de la poliquetofauna bentónica intermareal y submareal somera (<30 metros) de las prospecciones en fiordos, canales e islas en el marco del Proyecto Biodiversidad de Aisén (2001-2003). Un total de 76 taxa diferentes correspondientes a 30 familias fueron registrados contabilizándose un total de 1.364 individuos. Las familias Spionidae y Nereididae presentaron la mayor riqueza específica con nueve y ocho especies respectivamente, siendo Platynereis australis (Schmarda, 1861) el poliqueto más dominante a lo largo de todas las campañas de muestreo. Se registran por primera vez para la región ejemplares de las familias Chrysopetalidae, Saccocirridae y Arenicolidae, mientras que para otras especies se incrementa su rango de distribución. La alta riqueza de especies de algunas localidades (e.g. Isla Kent) acompañada de una serie de condiciones ambientales, oceanográficas y geográficas particulares hacen suponer la presencia de "hotspots" de diversidad en la región de Aisén. Se plantea la hipótesis que la alta abundancia y ocurrencia de ciertas especies de poliquetos respecto de otros invertebrados junto a condiciones específicas del ambiente en donde habitan permitirían generar la existencia de un "subiotopo" en los ambientes costeros estudiados caracterizado principalmente por la dominancia de estas especies de poliquetos.

Palabras clave: Polychaeta, intermareal, agua someras, biodiversidad.

\section{INTRODUCCIÓN}

La región de Aisén (43 85’ S - 48 90’ S), al sur de Chile, es una extensa zona de canales, fiordos e islas que presenta un particular interés científico debido a su aislamiento geográfico, pristinidad, endemismo (Moreno et al. 2006) y por encontrarse entre dos zonas biogeográficas (Brattström \& Johanssen 1983, Lancellotti \& Vásquez 1999). A pesar de las peculiaridades de la región, existen pocos estudios sobre flora y fauna marina; debido a su compleja geografía, conectividad y lejanía; aunados a las condiciones climáticas y oceanográficas adversas. El desconocimiento es aún mayor en cuanto a los invertebrados bentónicos (Reid \& Osorio 2000, John et al. 2003, Lancelotti \& Váquez 2000, Cañete et al. 1999 y Rozbaczylo \& Martínez 2003), por ello la relevancia de este estudio.

En los últimos 15 años, a partir de los cruceros oceanográficos CIMAR Fiordos coordinados por el Comité Oceanográfico Nacional de la Armada de Chile se han generado antecedentes e información de importantes grupos zoológicos tanto para la columna de agua como para los fondos marinos blandos ( $>20$ metros) (Silva \& Palma 2006), quedando todavía pendiente el estudio de las comunidades intermareales conformados por algas e invertebrados bentónicos.

Precisamente y en el contexto del conocimiento biológico de esta zona austral es que se realizó entre los años 1999 y 2003 el Proyecto Biodiversidad de Aisén. Esta iniciativa desarrolló diversas líneas de investigación destacándose la de "Biotopos Marinos", la que tuvo entre sus objetivos el estudio de la flora y fauna de ambientes costeros en Parques y Reservas marinas. La información biológica obtenida fue posteriormente utilizada como herramienta para el manejo y administración de las Aéreas Silvestres Protegidas de la región (Ramírez et al. 2002 ${ }^{1}$, John et al. 2002).

Las expediciones estivales realizadas permitieron obtener colecciones representativas de invertebrados marinos, desde la zona intermareal y submareal somera; principalmente moluscos, crustáceos, equinodermos, celenterados y poliquetos (Ramírez et al. 2002). Precisamente fue la clase Polychaeta, debido a su alta presencia en los ambientes estudiados, motivo de un análisis mayor.

En este mismo aspecto Cañete et al. (1999), consideran que los poliquetos bentónicos son un grupo muy diverso y abundante en fondos someros de fiordos y canales de la región de Aisén, sin embargo, su desconocimiento es bastante alto existiendo pocos estudios y trabajos publicados que demuestren su importante presencia y dominancia (Rozbaczylo et

1 Ramírez, M. E., P. Báez, S. Letelier \& E. Soto. 2002. Informe Biotopos Marinos Operación Raleigh 2001, 22 págs. 
al. 1997, Montiel 2005). Sólo algunas publicaciones de antigua data originadas a partir de importantes expediciones científicas dan cuenta de ello (WesenbergLund 1962, Hartmann-Schröder 1965, Ehlers 1901) de ahí la necesidad de profundizar y actualizar su conocimiento con la posibilidad de ampliar algunos registros o de encontrar nuevas especies.

Sólo en las últimas dos décadas la poliquetofauna submareal de fondos blandos ( $>20$ metros) ha tenido una mayor atención en la región con una serie de trabajos relacionados principalmente con estudios de composición y abundancia (Rozbaczylo et al. 2005, Rozbaczylo et al. 2006a, Rozbaczylo et al. 2006b; Montiel et al. 2004), biogeografía (Cañete et al. 1999) y endemismo (Montiel \& Rozbaczylo 2009). No obstante la zona intermareal, tanto de fondos duros como blandos, continúa escasamente explorada sin una estimación preliminar de su diversidad, composición y abundancia.

El objetivo de esta investigación es presentar los resultados de composición, riqueza y abundancia de la poliquetofauna bentónica intermareal y submareal somera obtenidos en diferentes sectores de la región de Aisén en el marco del proyecto Biodiversidad de Aisén.

\section{MATERIALES Y MÉTODOS}

\section{ZONA DE ESTUDIO Y \\ COBERTURA TEMPORAL}

Los poliquetos bentónicos fueron recolectados en tres diferentes campañas estivales (Enero a Abril) realizadas en los años 2001, 2002 y 2003. Durante el año 2001 la zona de estudio se ubicó en torno a los canales Martínez y Messier e islas

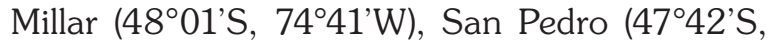
$\left.74^{\circ} 52^{\prime} \mathrm{W}\right)$ y Wager (4742'S, $\left.74^{\circ} 54^{\prime} \mathrm{W}\right)$, principalmente y zonas aledañas. Todos estos lugares en la Reserva Nacional Katalalixar $\left(48^{\circ} \mathrm{S}, 75^{\circ} \mathrm{W}\right.$ ) al sur de la región de Aisén. Durante el año 2002 la zona de estudio correspondió principalmente a las islas

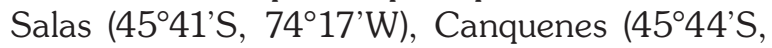
$\left.74^{\circ} 05^{\prime} \mathrm{W}\right)$, Kent $\left(45^{\circ} 09^{\prime} \mathrm{S}, 7^{\circ} 15^{\prime} \mathrm{W}\right)$ y Melchor (4509'S, $\left.74^{\circ} 09^{\prime} \mathrm{W}\right)$. Todas ellas situadas en la Reserva Nacional Las Guaitecas (RNLG), sector noroeste de la región de Aisén. Finalmente durante el año 2003 los muestreos estuvieron concentrados en el Fiordo Quitralco (454ㅇ's, $73^{\circ} 13^{\prime} \mathrm{W}$ ) y en isla Salas (4541'S, $\left.74^{\circ} 17^{\prime} \mathrm{W}\right)$ (RNLG) (Fig. 1). Detalle y posición de todos los sitios de muestreo y las localidades a las que pertenecen se presentan en la Tabla 1 .

TABLA 1. Estaciones de muestreo, localidad, posición, tipo de ambiente y forma de recolección pertenecientes a la poliquetofauna bentónica intermareal y submareal somera de la región de Aysén.

\begin{tabular}{|c|c|c|c|c|c|}
\hline Estación & Lugar & Latitud (S) & Longitud (W) & Fecha & Ambiente \\
\hline 1 & I. Millar, sector sur & $48^{\circ} 01^{\prime} 57^{\prime \prime}$ & $74^{\circ} 41^{\prime} 22^{\prime \prime}$ & $10 / 2 / 2001$ & $\begin{array}{c}\text { Intermareal } \\
\text { Submareal Rastra }\end{array}$ \\
\hline 2 & I. Millar, sector sur & $48^{\circ} 01^{\prime} 44^{\prime \prime}$ & $74^{\circ} 41^{\prime} 41^{\prime \prime}$ & $10 / 2 / 2001$ & $\begin{array}{c}\text { Intermareal } \\
\text { Submareal Macrocystis }\end{array}$ \\
\hline 3 & I. Millar & $48^{\circ} 01^{\prime} 45^{\prime \prime}$ & $74^{\circ} 41^{\prime} 34^{\prime \prime}$ & $13 / 2 / 2001$ & Submareal \\
\hline 4 & Canal Messier & $4751^{\prime} 16^{\prime \prime}$ & $74^{\circ} 59^{\prime} 51^{\prime \prime}$ & $14 / 2 / 2001$ & Submareal Rastra \\
\hline 5 & Canal Messier & $4755^{\prime} 40^{\prime \prime}$ & $75^{\circ} 07^{\prime} 10^{\prime \prime}$ & $16 / 2 / 2001$ & $\begin{array}{l}\text { Intermareal } \\
\text { Submareal }\end{array}$ \\
\hline 6 & I. Canquenes & $45^{\circ} 44^{\prime} 53^{\prime \prime}$ & 7403' 51'” & $2 / 3 / 2002$ & Intermareal \\
\hline 7 & I. Canquenes & $45^{\circ} 45^{\prime} 55,8^{\prime \prime}$ & $74^{\circ} 0443,3^{\prime \prime}$ & $3 / 3 / 2002$ & Intermareal \\
\hline 8 & I. Canquenes & $45^{\circ} 43^{\prime} 59,2^{\prime \prime}$ & $74^{\circ} 0520,4^{\prime \prime}$ & $4 / 3 / 2002$ & Intermareal \\
\hline 9 & I. San Pedro, sector sur & $47^{\circ} 42^{\prime} 39^{\prime \prime}$ & $74^{\circ} 52^{\prime} 65^{\prime \prime}$ & $6 / 3 / 2001$ & Intermareal arenoso \\
\hline 10 & I. Wager & $47^{\circ} 42^{\prime} 46^{\prime \prime} \mathrm{S}$ & $74^{\circ} 54^{\prime} 33^{\prime \prime}$ & $8 / 3 / 2001$ & $\begin{array}{c}\text { Intermareal } \\
\text { Arena fina (Corer) } \\
\text { Submareal Rastra }\end{array}$ \\
\hline 11 & I. San Pedro, sector norte & $47^{\circ} 41^{\prime} 92 ” \mathrm{~s}$ & $74^{\circ} 52^{\prime} 60^{\prime \prime}$ & $10 / 3 / 2001$ & $\begin{array}{c}\text { Intermareal } \\
\text { Submareal Rastra }\end{array}$ \\
\hline 12 & I. Riveros & $45^{\circ} 44^{\prime} 00,3^{\prime \prime}$ & $74^{\circ} 18^{\prime} 16,7^{\prime \prime}$ & $9 / 3 / 2002$ & $\begin{array}{l}\text { Intermareal rocoso } \\
\text { Submareal, Buceo }\end{array}$ \\
\hline 13 & I. Salas & $45^{\circ} 41^{\prime} 17,9^{\prime \prime}$ & $74^{\circ} 20^{\prime} 04,7^{\prime \prime}$ & $10 / 3 / 2002$ & Intermareal \\
\hline 14 & I. Salas & $45^{\circ} 43^{\prime} 63,1^{\prime \prime}$ & $74^{\circ} 16^{\prime} 44,8^{\prime \prime}$ & $11 / 3 / 2002$ & $\begin{array}{c}\text { Submareal } \\
\text { Rastra }\end{array}$ \\
\hline
\end{tabular}




\begin{tabular}{|c|c|c|c|c|c|}
\hline 15 & I. Prieto (Faro) & $45^{\circ} 41^{\prime} 17,9^{\prime \prime}$ & $74^{\circ} 20^{\prime} 04,7^{\prime}$ & $11 / 3 / 2002$ & $\begin{array}{l}\text { Intermareal } \\
\text { Submareal }\end{array}$ \\
\hline 16 & $\begin{array}{c}\text { I. Kent } \\
\text { (Estero Las Barracas) }\end{array}$ & $45^{\circ} 06^{\prime} 48,0^{\prime \prime}$ & $74^{\circ} 19^{\prime} 28,0^{\prime \prime}$ & $21 / 3 / 2002$ & Intermareal \\
\hline 17 & I. Kent & $45^{\circ} 05^{\prime} 36,8^{\prime \prime}$ & $74^{\circ} 15^{\prime} 56,2^{\prime \prime}$ & $22 / 3 / 2002$ & Intermareal \\
\hline 18 & $\begin{array}{c}\text { I. Kent } \\
\text { (Pto. María Isabel) }\end{array}$ & $45^{\circ} 04^{\prime} 13,5^{\prime \prime}$ & $74^{\circ} 17^{\prime} 07,2^{\prime \prime}$ & $24 / 372002$ & Intermareal \\
\hline 19 & $\begin{array}{c}\text { I. Kent } \\
\text { (Ensenada Río Coche) }\end{array}$ & $45^{\circ} 04^{\prime} 18,1^{\prime \prime}$ & $74^{\circ} 23^{\prime \prime} 00,5^{\prime \prime}$ & $25 / 3 / 2002$ & Intermareal \\
\hline 20 & I. Kent & $45^{\circ} 08^{\prime} 28,3^{\prime \prime}$ & $74^{\circ} 19^{\prime} 18,7^{\prime \prime}$ & $25 / 3 / 2002$ & $\begin{array}{c}\text { Intermareal } \\
\text { Submareal Rastra }\end{array}$ \\
\hline 21 & I. James & $45^{\circ} 01^{\prime} 13,7^{\prime \prime}$ & $74^{\circ} 21^{\prime} 10,7^{\prime \prime}$ & $26 / 3 / 2002$ & Intermareal \\
\hline 22 & I. Kent & $45^{\circ} 09^{\prime} 02,7^{\prime \prime}$ & $74^{\circ} 15^{\prime} 30,0^{\prime \prime}$ & $29 / 3 / 2002$ & Intermareal \\
\hline 23 & I. Kent & $45^{\circ} 09^{\prime} 48,3^{\prime \prime}$ & $74^{\circ} 17^{\prime} 52,3^{\prime \prime}$ & $30 / 3 / 2002$ & Intermareal \\
\hline 24 & $\begin{array}{c}\text { I. Melchor } \\
\text { (Estero San Eugenio) }\end{array}$ & $45^{\circ} 08^{\prime} 12,8^{\prime \prime}$ & $74^{\circ} 02^{\prime} 10,7^{\prime \prime}$ & $1 / 4 / 2002$ & $\begin{array}{l}\text { Intermareal } \\
\text { Submareal }\end{array}$ \\
\hline 25 & $\begin{array}{c}\text { I. Melchor } \\
\text { (Estero San Eugenio) }\end{array}$ & $45^{\circ} 09^{\prime} 07,8^{\prime \prime}$ & $74^{\circ} 03^{\prime} 29,1^{\prime \prime}$ & $1 / 4 / 2002$ & Intermareal \\
\hline 26 & I. Melchor & $45^{\circ} 09^{\prime} 59,9^{\prime \prime}$ & $74^{\circ} 09^{\prime} 12,1^{\prime \prime}$ & $2 / 4 / 2002$ & Intermareal \\
\hline 27 & I. Melchor & $45^{\circ} 10^{\prime} 21,3^{\prime \prime}$ & $74^{\circ} 09^{\prime} 11,0^{\prime \prime}$ & $3 / 4 / 2002$ & $\begin{array}{c}\text { Intermareal } \\
\text { Submareal Rastra }\end{array}$ \\
\hline 28 & Estuario Quitralco & $45^{\circ} 39^{\prime} 37,7^{\prime \prime}$ & $73^{\circ} 09^{\prime} 37,6^{\prime \prime}$ & $27 / 2 / 2003$ & $\begin{array}{c}\text { Submareal } \\
\text { Rastra }\end{array}$ \\
\hline 29 & $\begin{array}{l}\text { Estuario Quitralco } \\
\text { Península Llanos }\end{array}$ & $45^{\circ} 40^{\prime} 43,1^{\prime \prime}$ & $73^{\circ} 13^{\prime} 54,8^{\prime \prime}$ & $1 / 3 / 2003$ & Intermareal \\
\hline 30 & Estuario Quitralco & $45^{\circ} 40^{\prime} 58,7^{\prime \prime}$ & $73^{\circ} 9^{\prime} 54,7^{\prime \prime}$ & $3 / 3 / 2003$ & Intermareal \\
\hline 31 & $\begin{array}{l}\text { Estuario Quitralco } \\
\text { Estero Ballenita }\end{array}$ & $45^{\circ} 35^{\prime} 32,8^{\prime \prime}$ & $73^{\circ} 16^{\prime} 20,1^{\prime \prime}$ & $4 / 3 / 2003$ & Intermareal \\
\hline 32 & Estuario Quitralco & $45^{\circ} 37^{\prime} 49,1^{\prime \prime}$ & $73^{\circ} 17^{\prime} 8,9^{\prime \prime}$ & $5 / 3 / 2003$ & $\begin{array}{c}\text { Intermareal arenoso } \\
\text { Macrocystis }\end{array}$ \\
\hline 33 & Estuario Quitralco & $45^{\circ} 37^{\prime} 58,9^{\prime \prime}$ & $73^{\circ} 17^{\prime} 8,0^{\prime \prime}$ & $5 / 3 / 2003$ & Intermareal \\
\hline 34 & $\begin{array}{l}\text { Estuario Quitralco } \\
\text { Sector oeste }\end{array}$ & $45^{\circ} 37^{\prime} 55,8^{\prime \prime}$ & $73^{\circ} 20^{\prime} 49,1^{\prime \prime}$ & $7 / 3 / 2003$ & Intermareal \\
\hline 35 & I. Salas & $45^{\circ} 47^{\prime} 50,5^{\prime \prime}$ & $74^{\circ} 17^{\prime} 48,5^{\prime \prime}$ & $22 / 3 / 2003$ & Submareal \\
\hline 36 & I. Salas & $45^{\circ} 48^{\prime} 03,2^{\prime \prime}$ & $74^{\circ} 17^{\prime} 20,5^{\prime \prime}$ & $22 / 3 / 2003$ & $\begin{array}{l}\text { Submareal } \\
\text { Rastra }\end{array}$ \\
\hline 37 & Frente a I. Salas & $45^{\circ} 48^{\prime} 35,9^{\prime \prime}$ & $74^{\circ} 19^{\prime} 00,0^{\prime \prime}$ & $30 / 3 / 2003$ & $\begin{array}{l}\text { Submareal } \\
\text { Draga }\end{array}$ \\
\hline 38 & I. Salas & $45^{\circ} 47^{\prime} 51,0^{\prime \prime}$ & $74^{\circ} 17^{\prime} 40,6^{\prime \prime}$ & $30 / 3 / 2003$ & $\begin{array}{l}\text { Intermareal } \\
\text { Submareal } \\
\text { Macrocystis }\end{array}$ \\
\hline
\end{tabular}

\section{OBTENCIÓN DE LAS MUESTRAS}

La obtención de poliquetos bentónicos consideró los sectores y áreas destinados al proyecto "Biotopos Marinos" en donde se realizaron estudios de zonación, recolección de biota, transectos intermareales y el mar contiguo donde también se obtuvieron muestras de fondo.

La metodología de muestreo consistió mayoritariamente en la recolección manual de especímenes desde cada sitio de muestreo abarcando la zona intermareal de sustrato duro y blando según correspondiese. En el caso de playas de arena se tomaron muestras de sedimento en forma manual con un corer de PVC de $20 \mathrm{~cm}$ de diámetro y $30 \mathrm{~cm}$ de alto, las que posteriormente fueron cernidas en tamices de $500 \mu \mathrm{m}$ para el análisis de poliquetofauna.

Adicionalmente en algunos sitios de muestreo se obtuvieron poliquetos desde frondas y grampones de algas pardas laminariales (e.g. Macrocystis pyrifera) recolectadas manualmente o mediante buceo autónomo.

Los poliquetos submareales ( $<30$ metros) fueron obtenidos con: a) una draga manual tipo 


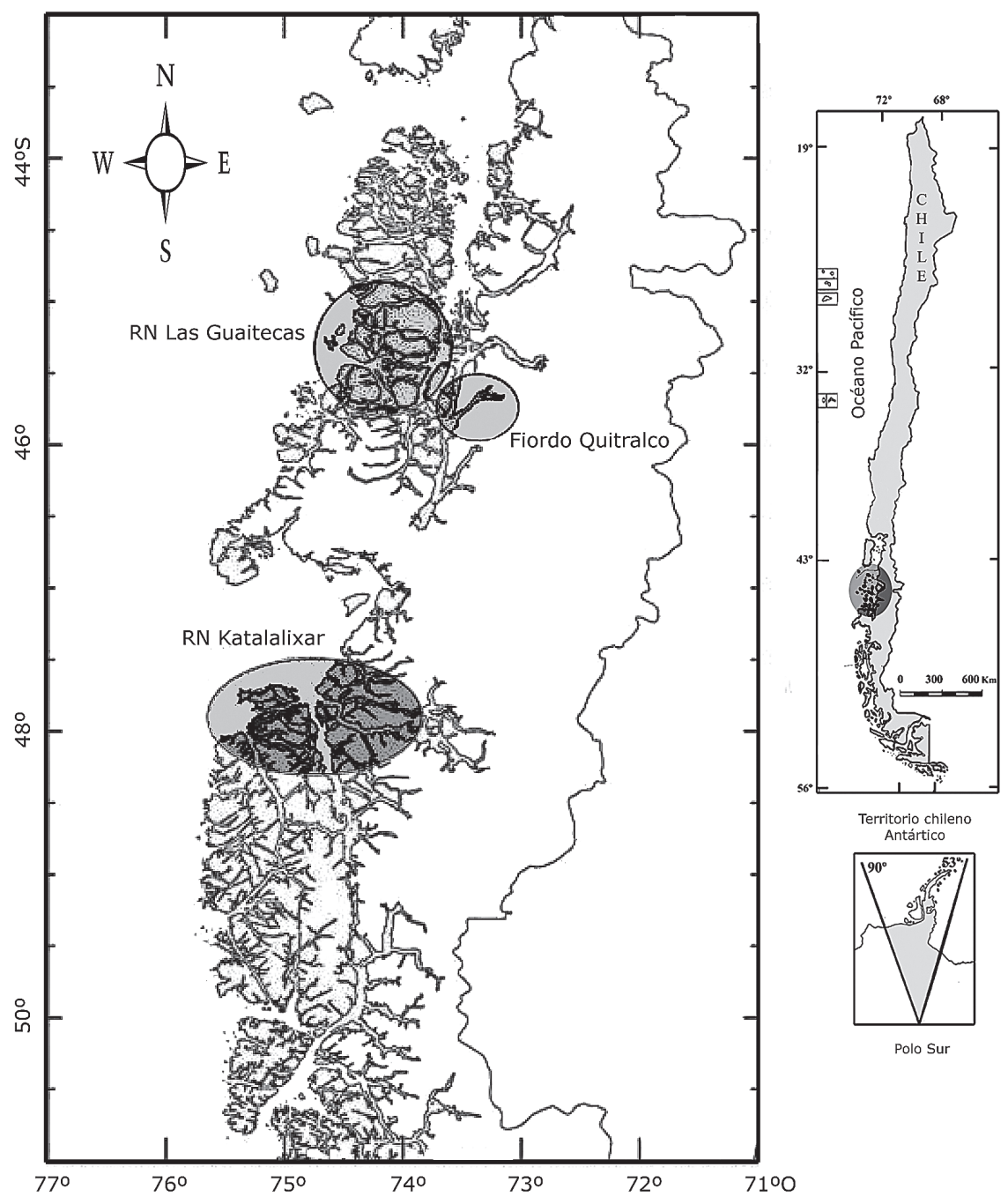

Fig. 1. Mapa de la región de Aisén, Patagonia Chilena. En círculos se señalan las zonas en donde se ubicaron los sitios de muestreo para la obtención de poliquetos bentónicos. RN: Reserva Nacional.

"Van Veen" de 0,01m² de area de mordida, b) una rastra tipo Agassiz de 50x20 $\mathrm{cm}$ de abertura de boca (arco metálico) y c) mediante buceo apnea y autónomo. En este último caso las muestras fueron recolectadas mediante corer de PVC (sedimento) o manualmente (agregaciones de mitílidos o grampones de algas pardas). Para el recuento total de muestras cada método y forma de muestreo fue considerada como una muestra individual y diferente.

Posteriormente cada muestra obtenida fue separada identificando a aquellos grandes grupos faunísticos presentes (Moluscos, Crustáceos, Equi- nodermos, Poliquetos, etc.). Los poliquetos fueron fijados en formalina al 14\% durante 48 horas para finalmente ser preservados en una solución de alcohol diluida en agua mar al 70\%, dentro de bolsas y frascos plásticos adecuadamente etiquetados para su identificación final.

Algunos parámetros ambientales de la columna de agua como temperatura, salinidad y $\mathrm{pH}$ fueron registrados en cada sitio de muestreo mediante un sensor Hanna Instruments previamente calibrado. Cada sitio de muestreo (estación) fue georreferenciada mediante GPS Garmin (Datum WGS 84). 


\section{IDENTIFICACIÓN DE LA POLIQUETOFAUNA}

Los poliquetos bentónicos fueron identificados con el apoyo de una lupa estereoscópica marca Nikon modelo SMZ800 y un microscopio Olympus CX31, ambos equipos ópticos con cámara clara y fotográfica acoplada. En los casos pertinentes y con el objeto de observar estructuras y caracteres morfológicos relevantes, se realizó disección manual y el empleo de tinción verde metilo.

Para la identificación de la poliquetofauna a nivel de familia se revisaron los trabajos de Rozbaczylo (1980), Fauchald (1977) y Rouse \& Pleijel (2001). Las identificaciones a nivel de género y especie fueron realizadas para cada caso consultando literatura especializada de la biblioteca del Museo de Historia Natural de Londres (NHM) así como trabajos clásicos relativos a la región (Hartman 1966, Wesenberg-Lund 1962; Hartmann-Schröder 1962 y 1965). Del mismo modo y para evitar errores en la identificación, se revisó material tipo del mismo Museo. Adicionalmente, algunas especies de poliquetos fueron corroboradas por los taxónomos Kristian Fauchald (Smithsonian Museum), Nicolás Rozbaczylo (P. Universidad Católica de Chile) y Juan I. Cañete (Universidad de Magallanes).

\section{RIQUEZA DE ESPECIES}

Con el único objetivo de comparar la diversidad de los lugares estudiados se calcularon los índices comunitarios de Riqueza de especies (S), Uniformidad de Pielou ( $\left.J^{\prime}\right)$, Rarefacción de Sanders (ES) y Diversidad de Shannon (H') utilizándose el software estadístico PRIMER v6 (Clarke \& Gorley 2006). Para ello se generó previamente una matriz de abundancia de especies versus localidades. Si bien existen diferencias en la metodología y número de muestras entre localidades, la presente comparación fue efectuada con el único propósito de destacar la alta diversidad de Isla Kent.

\section{RESULTADOS}

Se recolectaron 1.364 ejemplares de poliquetos bentónicos en 15 localidades distintas correspondientes a 38 sitios de muestreo (estaciones) y 62 muestras, desde la zona intermareal y fondos someros de fiordos, islas y canales de la región de
Aisén entre los años 2001 y 2003. Un total de 76 taxa diferentes pertenecientes a 30 familias fueron registrados, de los cuales 58 fueron identificados a nivel de especie y 18 preliminarmente a nivel de familia.

La composición taxonómica registra diferencias entre localidades y años de muestreo. En este sentido familias con alta riqueza de especies como Terebellidae, Phyllodocidae, Maldanidae, Goniadidae y Capitellidae no son registradas durante el año 2001 en la Reserva Nacional Katalalixar, al sur de la región de Aisén. Mientras que otras familias como Pectinaridae, Sabellaridae, Sabellidae, Scalibregmatidae, Eunicidae, Arenicolidae, Ophelidae, Saccocirridae y Paraonidae, entre otras, tiene registro único en una campaña de muestreo (año).

Las familias con mayor riqueza de especies fueron Spionidae con nueve y Nereididae con ocho, seguidas de Terebellidae y Polynoidae ambas con cinco especies. Nereididae fue la familia que registró el mayor número de individuos a lo largo de todo el estudio con 709 ejemplares, correspondiendo a un $52 \%$ del total de la poliquetofauna estudiada (Tabla 2).

En este sentido es importante destacar a Platynereis australis (Schmarda, 1861), poliqueto que con 423 ejemplares (31\% del total) representa la especie más abundante del presente estudio. Esta especie fue registrada con frecuencia en macroalgas intermareales y grampones de Macrocystis pyrifera. En torno a este hábitat destaca también la alta presencia de otros neréidos, ejemplares del género Aglaophamus (Nephtyidae) y poliquetos de la familia Polynoidae.

Por otra parte destacan los registros de las familias Chrysopetalidae, Arenicolidae y Saccocirridae no documentados antes para la región. Respecto de esta última familia no existen antecedentes ni registros formales descritos para la zona sur y austral de Chile (N. Rozbaczylo, Com. Pers.).

Se registran por primera vez para la región de Aisén los espiónidos Boccardia proboscidea Carrasco, 1974 y Scolelepis chilensis (HartmannSchröder, 1962); los neréidos Nereis pelagica Linnaeus, 1758, Perinereis falklandica (Ramsay, 1914) y Pseudonereis variegata (Grube, 1857) y el terebélido Streblosoma antarctica Monro, 1936, ampliándose el rango de distribución de estas especies. 
TABLA 2. Composición y abundancia de la poliquetofauna bentónica intermareal y submareal somera de la región de Aisén, XI región entre los años 2001 y 2003.

\begin{tabular}{|c|c|c|c|c|}
\hline \multirow[t]{2}{*}{ Especie } & \multirow[t]{2}{*}{ Familia } & \multicolumn{3}{|c|}{$\begin{array}{c}\text { Abundancia } \\
\left.\text { ( } \mathrm{N}^{\circ} \text { total de individuos }\right)\end{array}$} \\
\hline & & 2001 & 2002 & 2003 \\
\hline Ampharete sp. & Ampharetidae & & 1 & \\
\hline Ampharetidae sp. 1 & Ampharetidae & 1 & 3 & 1 \\
\hline Abarenicola sp. & Arenicolidae & & 1 & \\
\hline Mediomastus sp. & Capitellidae & & 5 & \\
\hline Capitella sp. & Capitellidae & & 16 & \\
\hline Capitellidae sp. 1 & Capitellidae & & 8 & 1 \\
\hline Capitellidae sp. 2 & Capitellidae & & & 4 \\
\hline Chaetopeturs variopedatus (Renier, 1804) & Chaetopteridae & & 2 & \\
\hline Chrysopetalidae sp. 1 & Chrysopetalidae & & 4 & \\
\hline Cirratulus sp. & Cirratulidae & & 9 & \\
\hline Cirriformia sp. & Cirratulidae & & 6 & \\
\hline Cirratulidae sp 2 & Cirratulidae & & 2 & \\
\hline Dorvillea sp. & Dorvilleidae & & 17 & \\
\hline Dorvilleidae sp.1 & Dorvilleidae & 1 & & \\
\hline Marphysa aenea (Blanchard, 1849) & Eunicidae & & 1 & \\
\hline Eunicidae sp.1 & Eunicidae & 1 & & \\
\hline Pherusa gymnopapillata Hartmann-Schröder, 1965 & Flabelligeridae & & 1 & \\
\hline Glycera sp. & Glyceridae & & 6 & \\
\hline Hemipodus sp. & Glyceridae & & 9 & 10 \\
\hline Glycinde armata (Kinberg, 1866) & Goniadidae & & 4 & \\
\hline Goniada sp. & Goniadidae & & 1 & \\
\hline Ophioglycera sp. & Goniadidae & & 3 & \\
\hline Gyptis sp. & Hesionidae & & 3 & \\
\hline Lumbrineris sp. & Lumbrineridae & & 12 & 4 \\
\hline Lumbrineridae sp. 1 & Lumbrineridae & & 4 & \\
\hline Euclymene sp. & Maldanidae & & 6 & \\
\hline Maldane sarsi antarctica Arwidsson, 1911 & Maldanidae & & 2 & \\
\hline Maldanidae sp. 1 & Maldanidae & & 1 & \\
\hline Maldanidae sp. 2 & Maldanidae & & 1 & \\
\hline Aglaophamus peruana (Hartman, 1940) & Nepthyidae & & 12 & \\
\hline Aglaophamus sp. & Nepthyidae & & 128 & 1 \\
\hline Nepthyidae sp.1 & Nephtyidae & 1 & & \\
\hline Namanereis quadraticeps (Blanchard, 1849) & Nereididae & & 20 & 73 \\
\hline Nereis callaona Grube, 1857 & Nereididae & & 2 & 22 \\
\hline Nereis pelagica Linnaeus, 1758 & Nereididae & 10 & & \\
\hline Perinereis falklandica (Ramsay, 1914) & Nereididae & & 2 & \\
\hline Perinereis vallata (Grube, 1857) & Nereididae & 8 & 24 & 59 \\
\hline Platynereis australis (Schmarda, 1861) & Nereididae & 153 & 250 & 20 \\
\hline Pseudonereis variegata (Grube, 1857) & Nereididae & & 4 & 1 \\
\hline Nereididae sp. 1 & Nereididae & & 61 & \\
\hline Saccocirrus sp. & Saccocirridae & & 3 & \\
\hline Ophelidae sp.1 & Ophelidae & & 1 & \\
\hline Leitoscoloplos kerguelensis (McIntosh, 1885) & Orbiniidae & & 1 & \\
\hline Leitoscoloplos sp. & Orbiniidae & 1 & 94 & \\
\hline Scoloplos sp. & Orbiniidae & & 4 & \\
\hline Aricidea sp. & Paraonidae & & 2 & \\
\hline Cistenides sp. & Pectinaridae & & & 1 \\
\hline
\end{tabular}


Eteone sp.

Eulalia sp.

Phyllodoce sp.

Phyllodocidae sp.1

Halosydna patagonica Kinberg, 1855

Harmothoe sp.

Hermadion maghalaensis Kinberg, 1855

Polynoidae sp. 1

Polynoidae sp. 2

Idanthysirus sp.

Branchioma sp.

Scalibregma sp.

Boccardia proboscidea Carrasco, 1974

Boccardia polybranquia (Haswell, 1885)

Boccardia sp.

Laonice sp.

Polydora sp.

Prionospio sp.

Rhincospio sp.

Scolelepis chilensis (Hartmann-Schröder, 1962)

Scolelepis sp.

Exogone sp.

Syllidae sp.1

Amphitrite sp.

Loimia sp.

Streblosoma cf antarctica Monro, 1936

Thelepus sp.

Terebellidae sp.1

Trichobranchus sp.

\begin{tabular}{|c|c|c|}
\hline Phyllodocidae & & 2 \\
\hline Phyllodocidae & & 2 \\
\hline Phyllodocidae & & 1 \\
\hline Phyllodocidae & & 1 \\
\hline Polynoidae & & 8 \\
\hline Polynoidae & 11 & 15 \\
\hline Polynoidae & & \\
\hline Polynoidae & & 62 \\
\hline Polynoidae & & \\
\hline Sabellaridae & & \\
\hline Sabellidae & & 1 \\
\hline Scalibregmatidae & & 1 \\
\hline Spionidae & & 28 \\
\hline Spionidae & 12 & \\
\hline Spionidae & & 2 \\
\hline Spionidae & & 7 \\
\hline Spionidae & & 16 \\
\hline Spionidae & & 3 \\
\hline Spionidae & & 2 \\
\hline Spionidae & 10 & \\
\hline Spionidae & & 2 \\
\hline Syllidae & & 11 \\
\hline Syllidae & 1 & 1 \\
\hline Terebellidae & & 1 \\
\hline Terebellidae & & 1 \\
\hline Terebellidae & & 4 \\
\hline Terebellidae & & 29 \\
\hline Terebellidae & & \\
\hline Trichobranchidae & & 1 \\
\hline
\end{tabular}

$\mathrm{N}^{\circ}$ total de individuos

Riqueza de familias

Riqueza de especies

Total número de sitios

Total número de muestras

En términos geográficos cabe hacer notar que la localidad de Isla Kent $\left(45^{\circ} 09^{\prime} \mathrm{S}, 74^{\circ} 15^{\prime} \mathrm{W}\right)$ en la Reserva Nacional Las Guaitecas, concentró más del $60 \%$ de la riqueza de especies de todo el estudio. En este lugar 46 especies de poliquetos fueron registradas correspondientes a 21 familias. La diversidad de Shannon $\left(\mathrm{H}^{\prime} \log _{10}\right)$ fue de 1,29 mientras que la rarefacción de Sanders $\left(\mathrm{ES}_{30}\right)$ registró un valor de 15,4 (Tabla 3)*

\section{DISCUSIÓN}

Se registraron poliquetos en la mayoría de los hábitats y ambientes desde los cuales se obtuvieron muestras. Por lo tanto, los organismos encontrados corresponden a especies que habitan sustratos blandos como duros y que abundan en sedimentos finos y gruesos, en los intersticios entre piedras y rocas, junto a algas y a distintos niveles de profundidad.

La composición taxonómica, riqueza de especies y de familias varía entre años y zonas estudiadas. Si bien existen algunos factores de orden geográfico (latitud) y oceanográfico (temperatura, salinidad) que podrían estar explicando estas variaciones, en concordancia a lo observado por Cañete et al. (1999) para el Fiordo Aisén y Puerto Cisnes, en este estudio las variaciones temporales y espaciales obedecen principalmente a las diferencias en el esfuerzo de muestreo registrado (número de estaciones y muestras). 
TABLA 3. Riqueza de especies (S), Uniformidad (J'), Rarefacción de Sanders (ES30), Diversidad $\left(\mathrm{H}^{\prime}\right)$ y Número total de muestras para las localidades estudiadas. EQ: Estero Quitralco.

\begin{tabular}{|c|c|c|c|c|c|}
\hline Localidad & $\mathrm{S}$ & J' & $\mathrm{ES}_{(30)}$ & $H^{\prime}\left(\log _{10}\right)$ & $\mathrm{N}^{\circ}$ muestras \\
\hline Isla Millar & 5 & 0,5304 & 4,614 & 0,3707 & 5 \\
\hline Canal Messier & 3 & 0,3625 & 2,802 & 0,1729 & 3 \\
\hline Isla San Pedro & 9 & 0,4515 & 5,738 & 0,4308 & 4 \\
\hline Isla Wager & 8 & 0,8282 & 8 & 0,7479 & 4 \\
\hline Isla Canquenes & 11 & 0,727 & 8,491 & 0,7571 & 3 \\
\hline Isla Riveros & 13 & 0,8715 & 11,37 & 0,9708 & 2 \\
\hline Isla Salas & 14 & 0,7814 & 10,21 & 0,8956 & 13 \\
\hline Isla Prieto & 14 & 0,6954 & 8,301 & 0,797 & 2 \\
\hline Isla Kent & 46 & 0,779 & 15,4 & 1,295 & 9 \\
\hline Isla James & 24 & 0,7278 & 11,21 & 1,005 & 1 \\
\hline Isla Melchor & 27 & 0,7925 & 12,72 & 1,134 & 6 \\
\hline Estero Quitralco & 15 & 0,6683 & 9,075 & 0,786 & 8 \\
\hline Península Llanos (EQ) & 12 & 0,729 & 8,735 & 0,7867 & 1 \\
\hline Estero Ballenita (EQ) & 5 & 0,7359 & 4,395 & 0,5144 & 1 \\
\hline
\end{tabular}

La mayor riqueza de especies observada para la familias Spionidae, Polynoidae, Terebellidae y $\mathrm{Ne}$ reididae y la abundancia registrada por Platynereis australis estaría explicada por la dominancia de macroalgas en la zona intermareal y de Macrocystis pyrifera en el submareal correspondiente a los sitios estudiados. $P$. australis y muchos neréidos forman agregaciones tubícolas entre macroalgas de la zona intermareal y son también comunes en grampones de algas pardas ( $M$. pyrifera) recolectados desde la zona submareal. Además habitan los sedimentos intermareales y submareales en donde se mezclan piedras, roca y arena creando un hábitat particular. Similares adaptaciones y estilos de vida fueron observados para especies de las familias Spionidae, Polynoidae y Terebellidae.

Esta distribución espacial supone la presencia de un "subiotopo" (John et al. 2003) caracterizado por la dominancia de algunas especies de poliquetos respecto de otros invertebrados como moluscos gastrópodos y crustáceos peracáridos (anfípodos e isópodos principalmente). En este caso la presencia de poliquetos neréidos como la especie Platynereis australis (Schmarda, 1861) podrán definir y caracterizar esta situación.

Sólo un adecuado estudio ecológico y poblacional orientado a conocer en el tiempo este ambiente, sus variables e interacciones, permitirán contrastar la hipótesis anterior y así confirmar o refutar la presencia de un hábitat particular o "subiotopo" propio de poliquetos bentónicos en la zona de canales e islas de la undécima región del sur de Chile.
Actualmente para la región de Aisén los registros de poliquetos intermareales y de fondos someros menores a 30 metros se concentran en sólo 4 publicaciones (Wesenberg-Lund 1962, Hartmann-Schröder 1962, 1965, y Cañete et al. 1999), contabilizándose un total de 57 especies (Rozbaczylo et al. 2005) para un número total indeterminado de estaciones y muestras, en la zona comprendida entre la boca del Guafo (4339'S) y el estero Elefantes (46 $\left.28^{\prime} \mathrm{S}\right)$.

En 1949, la "Lund University Chile Expedition, 1948-1949" (Wesenberg-Lund, 1962) registró 15 especies de poliquetos en un total de 9 estaciones, entre isla Guafo (433' 3 ) y canal Errázuriz, faro islote Diego (4539'20"S), en profundidades de 0-25 m. En 1962, Hartmann-Schröder, informa 7 especies de poliquetos, en isla James e isla Melchor, asociadas con Macrocystis pyrifera. En 1965, Hartmann-Schröder, da a conocer 20 especies de poliquetos en el archipiélago de los Chonos (isla James, puerto Aguirre, isla Melchor), asociadas a bancos de mitílidos. Mientras que Cañete et al. (1999), informan sobre poliquetos recolectados en 3 localidades submareales someras del fiordo Aisén (bahía Acantilada, desembocadura de río Cóndor y desembocadura de río Cuervo), registrando un total de 38 especies de las cuales sólo 25 fueron determinadas a nivel específico.

En la presente investigación 76 especies han sido distinguidas en el área comprendida entre el archipiélago de Los Chonos (RNLG), fiordo Quitralco y el sector del golfo de Penas (canal Messier e isla Millar); que corresponden a 38 estaciones y 62 mues- 
tras. Si bien esta riqueza de especies es mayor a la registrada por todos los estudios antes mencionados (57 especies), debe considerarse que en la presente investigación la determinación a nivel específico de muchas de ellas está en proceso. Adicionalmente no existe claridad en el número total de muestras respecto de los estudios anteriores y las metodologías para la toma de muestras han sido distintas en ambos sentidos. Por lo tanto comparaciones en ambos sentidos deben ser cuidadosas.

Con relación a la riqueza de familias no existe información publicada respecto del número total presente a la fecha para ambientes intermareales $y$ fondos someras inferiores a 30 metros. Sin embargo Rozbaczylo \& Martínez (2003) señalan que un total de 32 familias han sido registradas tanto para ambientes someros como profundos. En este sentido el presente trabajo constituye un aporte para el conocimiento del grupo (Polychaeta, Annelida) al registrarse un total de 30 familias y considerando que las familias Chrysopetalidae, Arenicolidae, y Saccocirridae constituyen primer registro para la región de Aisén.

En este sentido el registro de ejemplares de Chrysopetalidae resulta interesante ya que no existen antecedentes de la presencia de esta familia en regiones australes de Chile. Por su parte el registro de ejemplares de Arenicolidae, si bien importante, no debiese sorprender ya que existen diversos registros del género Abarenicola Wells tanto para Chiloé (Wells 1963) como para la región de Magallanes (Wells 1963, Ehlers 1897 y 1901). Por su parte, Moreno et al. (2007) indica que Abarenicola affinis chilienis Wells se sitúa en torno a los $42^{\circ} \mathrm{S}$, mientras que otras dos especies de este género habitarían entre los $53^{\circ}$ y $55^{\circ} \mathrm{S}$ (Provincia Magallanica). Posiblemente el no registro de esta familia anteriormente se debería a lo poco estudiado que se encuentra la zona intermareal en esta región. Finalmente el registro de 3 ejemplares del género Saccocirrus Bobretzky (Saccocirridae) amplían considerablemente el rango de distribución del género y de la familia ya que sólo se tenían antecedentes de esta familia para la tercera región del norte de Chile (Lee \& Correa 2004).

En cuanto a la composición taxonómica gran parte de los poliquetos determinados a nivel específico son anteriormente registrados para otras localidades de la región por diferentes autores y en ambientes intermareales como de fondos someros. Wesenberg-Lund (1962) da cuenta de Perinereis vallata, Glycinde armata y Marphysa aenea. Por su parte Cañete et al. (1999) informa en aguas del Fiordo Aisén la presencia de Platynereis australis, Namanereis quadraticeps, Aglaophamus peruana, G. armata y Leitoscoloplos kerguelensis. Mientras que Hartmann-Schröder (1965), señala el registro de Halosydna patagonica, Pherusa gymnopapillata, P. vallata y Boccardia polybranquia.

A profundidades mayores a 30 metros son además registrados Nereis callaona (Rozbaczylo et al. 2005), Maldane sarsi antarctica, Chaetopterus variopedatus (Rozbaczylo et al. 2006b) y Hermadion maghalaensis (Ehlers 1897).

En la presente investigación se registran por primera vez para la región los espiónidos Boccardia proboscidea Carrasco, 1974 y Scolelepis chilensis (Hartmann-Schröder 1962); los neréidos Nereis pelagica Linnaeus 1758, Perinereis falklandica (Ramsay 1914) y Pseudonereis variegata (Grube 1857) y el terebélido Streblosoma antarctica Monro 1936. Con ello se subraya la importancia de continuar y aumentar las investigaciones de la zona intermareal y submareal somera en la región de Aisén (<30 metros), contribuyendo de esta forma a complementar el conocimiento de la poliquetofauna bentónica de ambientes costeros y además a mejorar la información respecto de los rangos de distribución geográfica y batimétrica de algunas especies.

Los resultados presentados demuestran la alta riqueza de especies que presenta la región de Aisén en términos de poliquetos bentónicos coincidiendo con lo expresado por Rozbaczylo \& Simonetti (2000). Más aún si se considera que durante el presente estudio las muestras provienen exclusivamente de la zona intermareal y de fondos inferiores a 30 metros de profundidad. Esta diversidad taxonómica coincide con lo expresado por Hernández et al. (2005) y Moreno et al. (2006). Estos autores señalan que procesos de tipo climático como tectónico incidirían en la formación de hábitats distintos con características oceanográficas únicas, generándose de esta forma "hotspots" de alta riqueza faunística como para el caso de los poliquetos bentónicos. En este sentido Isla Kent, en la Reserva Nacional Guaitecas, presentaría rasgos de esta característica (hotspot) al concentrar más de un 60\% de las especies registradas durante el año 2002 y presentar valores de diversidad comparativamente mayores respecto del resto de las localidades investigadas (Tabla 3). 


\section{AGRADECIMIENTOS}

Los autores agradecen a la coordinación del Proyecto Biodiversidad de Aisén, en especial a Daniela Castro (CONAF) y Rich Howard (Raleigh International) por la asistencia en las campañas de terreno realizadas. También a todos aquellos que participaron en la recolección y separación de muestras desde diferentes ambientes durante la realización de las distintas fases de terreno. En especial a Andrew Miller (British Antarctic Survey); Tim Ferrero, David John, Nick Evans, Andrew Cabrinovich, Claire Valentine y Mary Spencer del Museo de Historia Natural de Londres y a Pedro Báez, Sergio Letelier y María Eliana Ramírez del Museo Nacional de Historia Natural de Santiago. Eulogio Soto agradece la beca otorgada por el Proyecto para realizar un Workshop taxonómico en el Museo de Historia Natural de Londres en Noviembre de 2002 con el propósito de profundizar la identificación de las especies encontradas. Los autores agradecen también a los profesores Nicolás Rozbaczylo, Juan I. Cañete y Kristian Fauchald por la colaboración en la identificación de algunas especies Iván Salinas (U. de Valparaiso) por los análisis estadísticos realizados y finalmente al Dr. Américo Montiel (Universidad de Magallanes) quien gentilmente nos ha invitado a participar de la realización de este volumen especial sobre poliquetos patagónicos.

\section{LITERATURA CITADA}

Brattström, H. \& A. Johanssen 1983. Ecological and regional zoogeography of the marine benthic fauna of Chile. Sarsia 68: 289-339.

Cañete, J. G. Leighton \& F. Aguilera 1999. Polychaetes from Aysén Fjord, Chile: distribution, abundance and biogeographical comparison with the shallow soft-bottom polychaete fauna from Antarctica and Magellan province. Scientia Marina 63 (Supl. 1): 243-252.

Clarke, K. R. \& R.N. Gorley. 2006. PRIMER v6: User Manual/Tutorial. PRIMER-E, Plymouth.

Ehlers, E. 1897. Polychaeten. Hamburger Magalhaenischen Sammelreise. Hamburg, 148 pp. 9 pls.

Ehlers, E. 1901. Die Polychaeten des magellanischenund chilenischen Strandes. Ein faunistischer Versuch. Festschrift zur Feier des Hundertfünfzigjährigen Bestehens der
Königlichen Gesellschaft der Wissenschaften zu Göttingen. (Abh. Math.-Phys. K.), Berlin, 232 pp., 25 pls.

Fauchald, K. 1977. The Polychaete Worms. Definitions and keys to the orders, families and genera. Natural History Museum of Los Angeles County, Science Series 28: 1-190.

Hartman, O. 1966. Polychaeta Myzostomidae and Sedentaria of Antarctica. Antarctic Research Series 7: 1-158.

Hartmann-Schröder, G. 1965. Zur Kenntnis des Sublitorals der chilenischen Küste unter besonderer Berücksichtigung der Polychaeten und Ostracoden. Tl. II. Die Polychaeten des Sublitorals. Mitteilungen aus dem Hamburgischen oologischen Museum und Institut 62 (Suppl.): 59-305.

Hernández, C. E., R. A. Moreno \& N. Rozbaczylo 2005. Biogeographical patterns and Rapoport's rule in southeastern Pacific benthic polychaetes of the Chilean coast. Ecography 28: 363-373.

John D.M., G.L.J. Paterson, N.J. Evans, M.E. Ramirez, M.E. Spencer Jones, P.D. Baez, T.J. Ferrero, C. A. Valentine \& D.G. Reid 2003. A Manual of Marine Biotopes of Region Aysen, Southern Chile. The Laguna San Rafael National Park, Estero Elefantes, Chonos Archipelago and Katalalixar. London, Biodiversity Aysen Project. 127 pp.

John, D. M., R. Foster-Smith, G. L. Paterson, M. E. Ramírez, N. J. Evans, M. E. Spencer Jones, D. G. Reid \& T. J. Ferrero 2002. A "Biotope" approach to the marine benthic biological assemblages of the Laguna San Rafael National Park, Chile. Boletín del Museo Nacional de Historia Natural 51: 159-173.

Lancellotti, D. A. \& J. Vásquez 1999. Biogeographical patterns of benthic macroinvertebrates in the Southeastern Pacific littoral. Journal of Biogeography 26: 1001-1006.

Lancellotti, D.A. \& J. Vásquez 2000. Zoogeografía de macroinvertebrados bentónicos de la costa de Chile: contribución para la conservación marina. Revista Chilena de Historia Natural 73(1): 99-129.

Lee, M.R. \& J.A. Correa 2004. Copper mine tailings disposal: consequences for the interstitial polychaete Saccocirrus sonomacus (Canalipalpata: Protodrilida). Journal of Marine 
Biological Association of the United Kingdom 84: 603-606.

Montiel, A. \& N. Rozbaczylo 2009. Distribución de los poliquetos de fondos blandos endémicos de fiordos y canales chilenos. Anales Instituto de la Patagonia 37 (1): 17-125.

Montiel, A. 2005. Biodiversity, zoogeography and ecology of polychates from the Magellan region and adjacent areas. Reports on Polar and Marine Research 505: 1-112.

Montiel, A., C. Ríos, E. Mutschke \& N. Rozbaczylo 2004. Poliquetos de fiordos y canales adyacentes al Campo de Hielo Patagónico Sur, Chile (Annelida: Polychaeta). Ciencia y Tecnología del Mar 27 (1): 49-67.

Moreno, R.A., C.E. Hernández. M. M. Rivadeneira, M. A. Vidal \& N. Rozbacylo 2006. Patterns of endemism in south-eastern Pacific benthic polychaetes of the Chilean coast. Journal of Biogeography 33: 750-759.

Moreno, R.A., N. Rozbaczylo, R.D. Sepúlveda, M.I. Marinkovic, W.E. Arntz \& S. Thatje 2007. On some ecological aspects of the lugworm Abarenicola affinis chiliensis Wells, 1963 (Polychaeta: Scolecida: Arenicolidae) from shallow soft bottoms of northern Chile. Helgoland Marine Research 61, 1-7.

Reid, D. \& C. Osorio 2000. The shallow-water marine Mollusca of the Estero Elefantes and laguna San Rafael, southern Chile. Bulletin of Natural History Museum London UK 66(2): 109-146.

Rouse, G. \& F. Pleijel 2001. Polychaetes. Oxford University Press. 354 pp.

Rozbaczylo, N. \& J. A. Simonetti. 2000. Diversity and distribution of Chilean benthic marine polychaetes: state of the art. Bulletin of Marine Science 67 (1): 359-372.

Rozbaczylo, N. \& S. Martínez 2003. Poliquetos bentónicos recolectados en el transcurso del crucero CIMAR 8 Fiordos en la región de Aysén, Chile (Annelida: Polychaeta). Informes preliminares Resultados Crucero CIMAR 8 Fiordos, 209-216.

Rozbaczylo, N. 1980. Clave para el reconocimiento de Familias de Anélidos Poliquetos del Mar Chileno. Studies on Neotropical Fauna and Environment 15: 167-196.

Rozbaczylo, N. 1985. Los anélidos poliquetos de Chile. Indice sinonímico y distribución geográfica de especies. Monografías Biológicas 3: 1-284.
Rozbaczylo, N. 2000. Indice bibliográfico sobre biodiversidad acuática de Chile: Poliquetos (Annelida: Polychaeta). Ciencia y Tecnología del Mar 23: 123-138.

Rozbaczylo, N., P. Vásquez, C. Maturana, R. A. Moreno \& E. Poulin 2008. Diversidad y caracterización genética de poliquetos bentónicos submareales, entre Boca del Guafo $\left(43^{\circ} 46^{\prime}, 40\right.$ S) y Golfo Elefantes ( $46^{\circ} 2^{\prime}, 00$ S), Chile, recolectados durante el crucero CIMAR 13 Fiordos (Annelida, Polychaeta). Informes Preliminares Crucero CIMAR 13 Fiordos 81-116.

Rozbaczylo, N., R.A. Moreno \& O. Díaz-Díaz 2005. Poliquetos bentónicos submareales de fondos blandos de la Región de Aysén, Chile: Clado Phyllodocida (Annelida, Polychaeta). Investigaciones Marinas 33 (1): 69-89.

Rozbaczylo, N., R.A. Moreno \& O. Díaz-Díaz 2006b. Poliquetos bentónicos submareales de fondos blandos de la región de Aysén, Chile: Clados Amphinomida, Eunicida, Spionida, Sabellida y Scolecida (Annelida, Polychaeta). Investigaciones Marinas 34 (1): 43-62.

Rozbaczylo, N., R.A. Moreno, O. Díaz-Díaz \& S. Martínez 2006a. Poliquetos bentónicos submareales de fondos blandos de la Región de Aysén, Chile: Clado Terebellida (Annelida: Polychaeta). Ciencia y Tecnología del Mar 29(1): 71-90.

Rozbazcylo. N, C. Ríos \& E. Mutsche 1997. Poliquetos de la Región de Magallanes: estado actual de su conocimiento a través de un análisis histórico y un estudio de caso. In: W. Arntz and C. Ríos (eds.) Abstracts presented in the International Congress "Marine Biological research in the Magellan region related to the Antartic", April 7-11, 1997, Punta Arenas, Chile.

Silva, N. \& S. Palma 2006. Avances en el conocimiento oceanográfico de las aguas interiores chilenas, Puerto Montt a cabo de Hornos. Comité Oceanográfico Nacional - Pontificia Universidad Católica de Valparaíso 162pp.

Wells, G.P. 1963. The lugworms of the southern cold temperate zone (Arenicolida, Polychaeta). Proceeding of Zoological Society of London 140: 121-159.

Wesenberg-Lund, E. 1962. Polychaeta Errantia. Reports of the Lund University Chile Expedition 1948-1949. (43). Lunds Universitets Årsskrift. N.F. Avd. 2, 57 (12): 1-139. 